\title{
Erst fragen, dann fahren!
}

\section{Carlos Beat Quinto}

Dr. med., Mitglied des FMH-Zentralvorstandes, Departementsverantwortlicher Public Health und Gesundheitsberufe

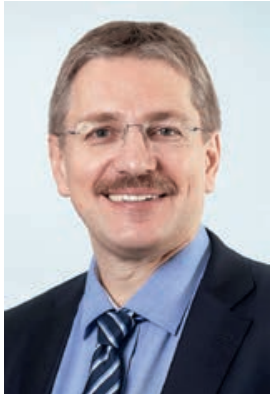

Was den eigenverantwortlichen Umgang von Fahrzeuglenkenden mit Medikamenten anbelangt, besteht in der Schweiz Nachholbedarf. Dies veranlasste die Beratungsstelle für Unfallverhütung, bfu, dazu, zusammen mit der FMH und pharmaSuisse eine Kampagne zum Thema «Fahren mit Medikamenten» zu lancieren. Ziel der Kampagne ist, Patientinnen und Patienten für die Problematik zu sensibilisieren und so die Anzahl von Unfällen zu senken. Ärztinnen und Ärzte sehen täglich unzählige Patientinnen und Patienten jeder Altersgruppe. Sie sind daher in der idealen Position, um Präventionsbotschaften zu platzieren und so einen Beitrag zur Gesundheitskompetenz zu leisten.

Fahren unter Medikamenteneinfluss stellt gemäss dem OECD-Bericht "Drogues au volant: Détection et dissuasion» ein unterschätztes, schwer fassbares Problem für die Sicherheit auf den Strassen dar. Anders als bei Alkohol handelt es sich bei Medikamenten um eine Vielzahl von Substanzen mit unterschiedlichsten Wirkungen, potentiellen Nebenwirkungen und Interaktionen [1]. Das Wissen über den Einfluss von Medikamenten auf die Fahrfähigkeit ist in der Bevölkerung weniger verankert als dies bei Alkoholkonsum der Fall ist. Rund 3500 registrierte Medikamente sind in der Schweiz zugelassen. Diese Medikamente können die Fahrfähigkeit beeinträchtigen, und

\section{Wir sind in der Lage, Patientinnen und Patienten auf motivierende Art kompetent und unabhängig zu beraten.}

zwar auch solche, die nicht rezeptpflichtig sind, und auch unabhängig des Alters der Person, welche die Medikamente einnimmt. Mögliche Folgen für die Fahrfähigkeit können verminderte Reaktionsfähigkeit, reduzierte Urteilsfähigkeit und Selbstkontrolle, Konzentrationsschwäche oder Sehstörungen sein. Als Ärztinnen und Ärzte sind wir in der Lage, Patientinnen und Patienten auf motivierende Art kompetent und unabhängig zu informieren und zu beraten und so zur Unfallverhütung beizutragen. Insbesondere bei der Einnahme von mehreren Medikamenten oder während Einschleich- oder Ausschleichphasen ist erhöhte Aufmerksamkeit gefragt. Zudem ist auf die Gefahr von Mischkonsum hinzuweisen. Gleichzeitiger Konsum von Alkohol oder anderen Substanzen mit Medikamenten kann unerwünschte Wirkungen noch verstärken.

Die Kampagne darf keine Angst vor Medikamenten machen. Denn zugleich gibt es Personen, die nur aufgrund der eingenommenen Medikamente ihre Fahrfähigkeit erhalten können - auch dies eine zentrale Botschaft der Kampagne. Ihr Ziel ist, das Wissen der Patientinnen und Patienten hinsichtlich Therapietreue und Fahrfähigkeit zu verbessern. Das bedingt auch, dass die Informationen zur medikamentösen Therapie, welche die Patientinnen und Patienten von ihrer Ärztin und Apothekerin erhalten, in die gleiche Rich-

\section{In der Schweiz sind rund 3500 registrierte} Medikamente zugelassen, die einen Einfluss auf die Fahrfähigkeit haben.

tung gehen. Widersprüchliche Informationen verunsichern Patientinnen und Patienten und gefährden die Therapietreue sowie das Kampagnenziel. So ist die Kampagne auch eine Chance für die Interprofessionalität - wohl wissend, dass sich die Ressourcenlage hierfür bei Ärzte- und Apothekerschaft sowie bei weiteren Medizinal- und Gesundheitsberufen seit dem zweiten Tarifeingriff laufend verschlechtert.

Um Ärzte- und Apothekerschaft, medizinische Praxisassistentinnen und Pharmaassistentinnen zu unterstützen, stellt die Beratungsstelle für Unfallverhütung, bfu, eine Vielzahl an Materialien kostenlos zur Verfügung: Plakate, zwei Videos, eine Internetseite, Flugblätter sowie Aufkleber für Medikamentenpackungen. Der Kampagnenstart ist für den 21. Juni 2018 vorgesehen, die Materialien können auf der Kampagnen-Webseite bestellt werden: www.fragen-dann-fahren.ch.

Literatur:

1 OECD. Drogues au volant: Détection et dissuasion. Editions OECD, Auflage 2010. http://www.oecd-ilibrary.org/transport/drogues-auvolant_9789282102787-fr. 